American Journal of Applied Sciences 8 (9): 843-847, 2011

ISSN 1546-9239

(C) 2011 Science Publications

\title{
Model Development for Health Promotion in the Elderly Participating in Communities
}

\author{
${ }^{1}$ Supat Jampawai, ${ }^{1}$ Somporn Pothinam, ${ }^{2}$ Manop Kanato, \\ ${ }^{1}$ Pramote Tongkrajai and ${ }^{1}$ Pitsamai Homjumpa \\ ${ }^{1}$ Department of Medicine, Faculty of Medicine, \\ Mahasarakham University, Maha Sarakham 44000, Thailand \\ ${ }^{2}$ Department of Community Medicine, Faculty of Medicine, \\ Khon Kaen University, Khon Kaen, Thailand
}

\begin{abstract}
Problem statement: The problem of the research derives from a lack of research study to develop the suitable model for health promotion in the elderly and examine factors concerning the health promotion in the elderly. This research aimed to study and develop suitable health promotion model for elderly participating in communities and examine factors related to the health promotion of the elderly. Approach: The samples were 654 older people. This study was participatory action research including many stages which were situation analysis, action and assessment. The explored location was the area of Nonsa-ad Sub-District, Nong Ruea District, Khon Kaen Province. Results: The results of the study led to the process of health promotion for the elderly participating in the communities in the action stage including the participation of the communities, local administrative organizations and government agencies consisting of health service centers and department of social development and human security to drive the thinking of the elderly and push it into real practice. The activities for the health promotion were health check-up, home visit of volunteers and public health staff, exercise, having suitable food, stress management, adequate sleep, not to drink alcohol, not to take steroid medicine, not to have betel nut, not to smoke, avoid accident and not to work hard. After 1 year of these activities, the health condition of the aged people was better while several health problems decreased such as the rate of sickness, risk group of chronic diseases, join and muscular pain. Besides, diabetes and blood pressure were at the same level. The patients with diabetes and blood pressure were able to better control glucose level and blood pressure at the safe rate without any complications. The whole quality of life of the elderly on physical and mental health, daily routine, society and finance were also better at $63.15 \%$. Furthermore, sex and age were significantly related to the health promotion for the elderly with $\mathrm{p}<0.0001$ and $\mathrm{p}<0.012$, respectively. However, religion, education and occupation were not significantly related to the health promotion. Conclusion/Recommendations: The aged persons should pay attention to their health first to learn appropriate treatment. Government organizations should take care of the budget continually to general people as well as the elderly to complete the target of the health promotion in contexts of community, tradition, beliefs, rituals and culture. These factors could help the aged persons live in the society and the environment properly to have strong physical and mental health with their warm families.
\end{abstract}

Key words: Model development, health promotion, elderly participating, ministry of public health, health care staffs, mental health, budget continually, chronic diseases

\section{INTRODUCTION}

Demographic transition generally happens around the world due to advances in medical and public health technology which can help extend human life and reduce birth rate. This situation affects change in global population structure. According to the world population information from United Nations in 2005, the population aged over 60 would increase from $10.3-20.4 \%$ in 2045 while the population aged over 65 would rise from 7.3$14.3 \%$ in 2040 (World Population 2007 United Nation). This data indicated that the total population would become an aged society in 2040. However, young population trend (aged newborn to 14) would decrease from $28.3 \%$ in $2005-20.4 \%$ in 2045. This detail represented that the global population structure would

Corresponding Author: Supat Jampawai, Department of Medicine, Faculty of Medicine, Mahasarakham University,

Tambon Talad, Muang District, Maha Sarakham 44000, Thailand 
Am. J. Applied Sci., 8 (9): 843-847, 2011

change from young to aged persons or aged society. In Thailand, a person who aged over 60 is regarded as an elderly. The population structure of Thailand is in the same direction as the global population structure. There were about 64.5 million people in Thailand during 2004 which demonstrated that Thailand had become to the aging society. According to analysis of National Statistical Office, it is expected that Thailand would approach the population aging. In 2024, the estimated population of Thailand would reach 70.5 million people and the aged persons over 60 would continue to grow at $20.5 \%$. As the above situation, the number of aged people is rising all the time which may cause future problems like developed countries. These issues include "expansion of economic system, social disability and health problem affecting the entire economic and social development". Government budget should be prepared for aged-care such as medical payment, health promotion and welfares because the aged persons are more likely to depend upon other people (Pylypa, 2009; Oakley and Marsden, 1984). To reduce the stated problems, it is necessary to develop a suitable and integrated model for health promotion in the elderly participating in communities (PRB, 2008). This model development aimed to help the aged people have appropriate health promotion and be able-bodied for proper daily selfhelp. It can declined social dependency of the elderly which can make both physical and mental health better and improve their quality of life also. For these reasons, it is very essential to find the suitable model for health promotion in the elderly participating in communities for better health and quality of life of the aged persons in Thailand.

\section{MATERIALS AND METHODS}

This research was a participatory action research including 6 processes within a research cycle. Some steps were interrelated while some procedures were done simultaneously. However, it had to stop an activity and then repeated it again or even developed such step continually at the same time:

Step 1: Gathering information. The author had to prepare everything before entering research area

Step 2: Finding problems and providing solutions. A plan to solve the health problems of the aged people was needed by seeking a proper type for health promotion, disease prevention and better living. This step required the data collected from step 1 to arrange project plan to create learning and participating process in the communities. The model development for health promotion in the elderly
Step 3: Operating the project plan. Every activity plan from step 2

Step 4: Data analysis. The data analysis would be done while operating the procedures. As stated above, the data analysis could be carried out in every step. The model analysis for the health promotion in the elderly would be completed after operation at various intervals to improve the activities. The data collected from interview, in-depth interview, participant observation, non-participant observation and investigation of usage provided services in the health center for the elderly

Step 5: Reflection. This step was to analyze all collected data and the activities of the health promotion in the elderly to find. It aimed to check which factor gave expected result and needed improvement. This information would be used for the reflection and continuous planning to solve new problem.

Step 6: Re-plan. When the new problem was found, there should be an adjustment. This process would run constantly like a cycle during research period. Informant group, anyway, was composed of community leaders, health care staffs or volunteers

Research tools: (1) Data collection tools: This study was a qualitative research using several data tools to collect qualitative data during whole period; Community participation of villagers, focus group discussion, observation, in-depth interview, brainstorming, multidimensional quality of life and health checkup instrument. (2) Quality investigation of research tools and data: The data reliability was tested by the triangulation; data triangulation, investigator triangulation and theory triangulation.

Data analysis: (1) Quantitative data ; was collected from the health checkup including blood glucose, weight, waistline, blood pressure and other examined factors to compare before and after results. Percentage, frequency and mean were applied to measure the quality of life of the aged persons in the rural area by the multidimensional quality of life instrument. Additional, Wilcoxon signed ranks test was utilized to consider the difference of the quality of life before and after the research for further discussion. (2) Qualitative data; gathered from field notes was analyzed by content analysis to summarize and adjust some steps in each process until the end of research duration. Consequently, there would be a conclusion to answer the research questions. 


\section{RESULTS}

The population in Nonsa-ad Sub-District, Nong Ruea District, Khon Kaen Province was around 10, $120 ; 4,318$ male and 5,802 female. Number of households including father, mother and children was 2,010 . The aged persons over 60 were 654 or $6.46 \%$ of total population. The results found that most of the elderly were farmers/agriculture $72.5 \%$ unemployed stay-at-home aged persons $21.9 \%$ and elementary occupations/laborer $21.9 \%$ because they still feel strong. Some of them were hired to transplant rice seedlings and sugarcane workers $2.1 \%$.

Most of young people would become job hunters in the city and neighboring factories after rice planting and sugarcane harvesting. Some worked full-time in the factories near their houses. The people in such area commuted round trip to work by minibuses for the factories' workers. Therefore, there was no problem with abandonment of domicile because they could work near their homes, stay with their families and look after the elderly also. Notwithstanding, some young people still worked in Bangkok and other provinces. They rarely backed home during cultural festivals such as the end of Buddhist Lent Day, New Year period or Song Kran Day (water festival). This group of people left the aged persons at home alone to bring up their young children studying in kindergartens or elementary schools. Several aged persons had to take care of teenage grandchildren which was regarded as quite hard responsibility for them. This burden might also affect the physical and mental health problem of the elderly.

Over $90 \%$ of the aged people were Buddhists and local temples and monks were their center of mind. Another place for making religious ritual was "Ta $\mathrm{Pu}$ ", a spirit shrine of the village. Every villager respected this holy shrine as their sacred item which they believed that the Ta Pu spirit would protect the village from bad things. The elderly $51.8 \%$ were young-old, $34.9 \%$ were mediumold and $13.3 \%$ were old-old. This data was concordant with domestic study indicating that the majority of elderly persons were between 60-69 years old, over half of total elderly population of the country. Most of them or $55.4 \%$ were married and widowed respectively. Besides, $40.5 \%$ of them lived with descendants while 18 or $2.8 \%$ of the aged persons were single.

According to data synthesis of Nonsa-ad SubDistrict, it found that the organizations concerning with the aged people care and the model development for health promotion in the elderly in the communities were Nonsa-ad Sub-district Administrative Organization, local government organizations such as representatives from Ministry of Public Health, Ministry of Social
Development and Human Security, Ministry of Interior and the communities. The process started from a meeting of the villagers on general problems in the communities to find out resolutions. One of the topics was related to allowance of the aged people, the health problems and living conditions. Ways to solve the problems of the elderly of 14 villages in Nonsa-ad SubDistrict were similar. For example, the lack of elderly caregivers was because some aged persons had no any descendant while some elderly suffered from poverty and economic problem. Some of them had chronic diseases such as diabetes, high blood pressure, backache, legs ache and joint pain. Moreover, certain aged persons had fatigue, loss of appetite, blurred vision, stone deaf, mental health problem from insomnia, anxiety on their own illness and descendants and difficulty in daily life that they had to depend on other people. Several elderly also faced the social and environmental problems affecting their peaceful way of life. They hoped to recover these symptoms and needed the health care staffs to help support the health promotion of the elderly in the villages.

To the resolution, the aged people established an elderly club of the village and appointed a president, a vice president, a secretary, a treasurer and members. In addition, the representatives from each village also joined the conference at the sub-district administration organization to plan the suitable model development for health promotion of the elderly participating in the communities. This assembly was operated by the aged people while the researcher and team gave them information and academic source. All decision was made within the elderly club. The operating budget support was provided by the sub-district administration and Social Development and Human Security of Khon Kaen. The commitment proposed that the sub-district administration should increase the elderly allowance and the sub-district administration agreed to rise the number of the elderly who received the allowance from 2-3 aged persons/village to 5 aged persons. Later, the government announced a policy to support the allowance to every aged person at 500 baht/month which was regarded as a successful intention of the elderly club. To the activities of health promotion, furthermore, the aged people agreed to participated training on the health promotion. They preferred learning which activity could make them healthy and how to have proper living as well as prevent the disease. The budget of this project was also given from the sub-district administration to provide knowledge on the health promotion and the suitable exercise. The elderly in each village practiced Yoga, wand exercise and other activities applied for the elderly groups. Each aged person would differently practice as much as they could with 
regard to their physical condition. They might exercise at home or anywhere in free time without any assembly. According to the social context, the elderly could not gather into a group because they had own responsibility to do. The caregivers, nevertheless, would assist disabled elderly and give the elderly relatives advice on treatment and static exercise with help from both the caregivers and the elderly relatives.

To the aged people with the chronic disease or too old for self-care, the elderly club requested for the budget to train the descendants or local health volunteers called "elderly care volunteers" supported by Provincial Social Development and Human Security to prepare 2-3 volunteers for each village, totally 43 persons. These caregiver duties were to correctly aid the elderly following the academic principles, visit the elderly in the villages and provide suggestions on caregiving at home which were very impressive for the aged persons. Additional, the elderly care volunteers also coordinated with the health care staffs and health care volunteers to offer basic health checkup in the communities to find the chronic disease and prevent any side effect. To the religious activities, the aged persons combined their groups together to be pillars of the communities to maintain local culture. They led the villagers and youth group to participate the religious rituals at the temple, for instance, Buddhist Lent Day, the end of Buddhist Lent Day and Lord Buddha Day. The elder group was also the leader of other ceremonies such as making offerings to the spirits, ordination and marriage. The communities supported and admired the aged persons to do these actions which could create shared value to the aged persons. Another impressive activity promoting gratitude was Songkran Day. Many descendants would pay respects to the elderly by pouring water for the elderly, asking for blessing and giving the elderly presents. This festival was held at the sub-district administration and in each village as well as within the villagers' families. Formerly this activity was not obvious but it was the activity which most of aged persons had been waiting for, according to the data analysis. The Songkran Festival was successfully arranged during this research and would be inherited afterward.

Besides, additional activities during this study were connected to the elderly. There were cremation fund in the village, community saving fund only at $1 \mathrm{baht} /$ day. The elderly club was run strongly as seen from budget request from the government, Sub-district Administrative Organization and communities, to prepare the activities for the communities as defined purposes. Then Wilcoxon Signed Ranks Test was used to analyze the data collected from all activity operation to identify the quality of life of the elderly.
Entire quality of life of elderly before and after the project ; The entire quality of life of elderly in Nonsaad Sub-District was summarized into total scores of 6 aspects of quality of life including daily routine, physical health, mental health, social relationship, environment and economic aspects.

The Wilcoxon Signed Ranks Test was applied to comparative analysis of the entire quality of life of the elderly due to abnormal distribution of the data. There was no significant difference between the quality of life before and after the research ( $\mathrm{p}$-value $=0.000$ ). Moreover, $65.90 \%$ of the elderly had better quality of life while $35.6 \%$ of them had low quality of life before the research. Nevertheless, $0.5 \%$ of the aged people had low quality of life after the research while $44.2 \%$ of them had medium quality of life as well as $8.6 \%$ had high quality of life before the research. The result also showed that $55.4 \%$ of the elderly had better quality of life after the research.

\section{DISCUSSION}

This study found new results which had never been revealed anywhere in Nonsa-ad District to support the health promotion for the elderly participating in the communities. Formerly, the health promotion for the elderly emphasized only hygiene knowledge and exercise methods which were ineffective for them. This research, anyway, discovered a fact that there were many ways to help the aged persons be healthy. It demanded several factors and community participation due to some limitations of the elderly such as chronic disease, physical and mental problem. Therefore, the aged people should be more understanding of health information and self limitations. They should have strong intention to strengthen their health also. Besides, the elderly should be supported to be the leaders of cultural and religious activities while local health experts or health care volunteers should provide knowledge to the people in the communities and the relatives of the aged persons. The elderly care volunteers should play more important role in risk screening to prevent the chronic disease and other effects of such disease by using medical instruments, for instance, blood pressure machine, blood glucose monitor and weight scale. There should be a visit to give advice on health care and proper exercise at home for those elderly who could not help themselves. These activities gave satisfactory results to let the people in the communities help each other which would become strength for the health promotion in the elderly. Also, it created a good relationship between the aged persons and other villagers (Jackson, 1994). The sub-district administrative organization should assist more budget 
to operate the activities related to the health promotion as well as coordinate with the government organizations, especially the health care centers and the hospitals, to prepare academic information and correct methods for better result in the health promotion. (Palank, 1991). Finally, to receive the effective outcome, the elderly must collaborate with their relatives closely meanwhile the health care staffs, the elderly health care volunteers and the subdistrict administrative organization must not neglect this issue. Sincerely, the government sector should give serious attention to the health promotion to achieve the better quality of life of the elderly (Intaranongpai et al., 2011).

\section{CONCLUSION}

The purposes of suitable model development for health promotion in the elderly participating in the communities were to help them have better physical and mental health for their ages to prevent the chronic diseases caused from weak physical and mental health. The aged persons should pay attention to their health first to learn appropriate treatment. They should be the center for health promotion suitably supported by the communities including their descendants, health care staffs and trained elderly health care volunteers. Moreover, the community leaders should assist sufficient welfare for the aged people while the monks should sustain the moral support and religious rituals of the elderly.

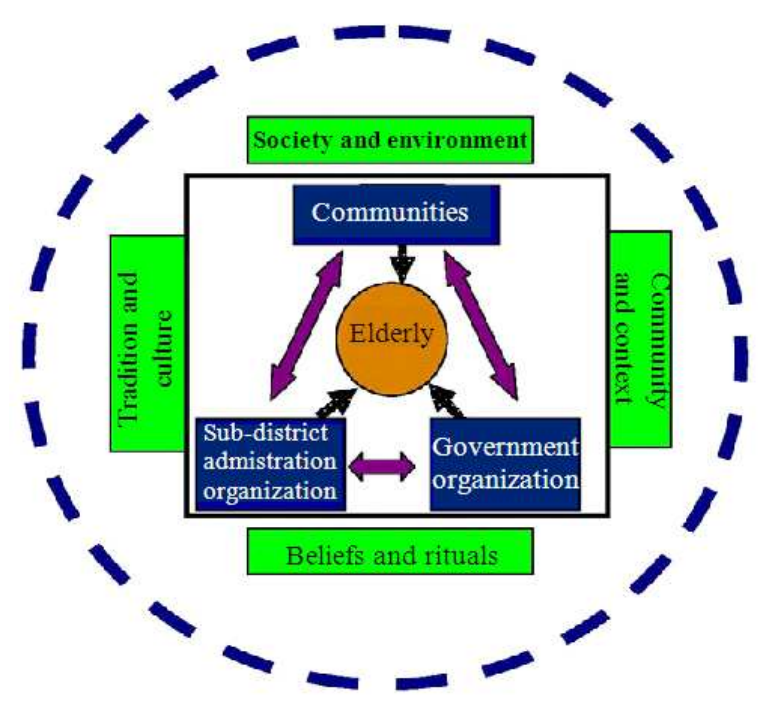

Fig. 1: The model of the health promotion in the elderly participating in the communities
The government organizations such as Ministry of Public Health, Ministry of Social Development and Human Security, Ministry of Interior and the subdistrict administrative organization should take care of the budget continually to general people as well as the elderly to complete the target of the health promotion in contexts of community, tradition, beliefs, rituals and culture. These factors could help the aged persons live in the society and the environment properly to have strong physical and mental health with their warm families. This could allow them to help themselves as it should be which would make them proud. The quality of life of the elderly was shown in Fig. 1.

The important components of the model consisted of three organization networks including: Sub-District Administrative Organization, Communities and Government Organizations.

\section{ACKNOWLEDGEMENT}

The researchers express their sincere appreciation and thanks for all of those who supported in one way or another.

\section{REFERENCES}

Intaranongpai, S., S. Pothinam, P. Tongkrajai and N. Sreenu, 2011. How does primary care move? An analytical evidence of management for accessibility and equity, Thailand. Eur. J. Soc. Sci., 21: 249-259.

Jackson, M.F., 1994. Discharge planning: Issues and challenges for gerontological nursing: A critique of the literature. J. Adv. Nurs., 19: 492-502. DOI: 10.1111/j.1365-2648.1994.tb01112.x

Pylypa, J., 2009. Elder authority and the situational diagnosis of diarrheal disease as normal infant development in Northeast Thailand. Qual. Health Res., $\quad$ 19: 965-675. DOI: 10.1177/1049732309338867

Oakley, P. and D. Marsden, 1984. Approach to participation in rural development. Geneva; WHO., pp: 31.

Oakley, P. and D. Marsden, 1984. Approaches to Participation in Rural Development. 1st Edn., International Labour Office, Geneva, ISBN9221035948, pp: 91.

Palank, C.L., 1991. Determinants of health-promotive behavior: A review of current research. Nurs Clin North Am., 26: 815-832. PMID: 1945937

PRB, 2008. World Population Data Sheet. Population Reference Bureau. 